\title{
Demi-linear duality
}

\author{
Ronglu Li $i^{* *}$, Aihong Chen ${ }^{1}$ and Shuhui Zhong ${ }^{2}$
}

\footnotetext{
*Correspondence: rongluli@yahoo. com.cn

'Department of Mathematics, Harbin Institute of Technology, Harbin 150001, P.R. China

Full list of author information is available at the end of the article
}

\begin{abstract}
As is well known, there exist non-locally convex spaces with trivial dual and therefore the usual duality theory is invalid for this kind of spaces. In this article, for a topological vector space $X$, we study the family of continuous demi-linear functionals on $X$, which is called the demi-linear dual space of $X$. To be more precise, the spaces with non-trivial demi-linear dual (for which the usual dual may be trivial) are discussed and then many results on the usual duality theory are extended for the demi-linear duality. Especially, a version of Alaoglu-Bourbaki theorem for the demilinear dual is established.
\end{abstract}

Keywords: demi-linear, duality, equicontinuous, Alaoglu-Bourbaki theorem

\section{Introduction}

Let $\mathbb{K} \in\{\mathbb{R}, \mathbb{C}\}$ and $X$ be a locally convex space over $\mathbb{K}$ with the dual $X^{\prime}$. There is a beautiful duality theory for the pair $\left(X, X^{\prime}\right)$ (see [[1], Chapter 8]). However, it is possible that $X^{\prime}=\{0\}$ even for some Fréchet spaces such as $L^{p}(0,1)$ for $0<p<1$. Then the usual duality theory would be useless and hence every reasonable extension of $X^{\prime}$ will be interesting.

Recently, $\mathcal{L}_{\gamma, U}(X, Y)$, the family of demi-linear mappings between topological vector spaces $X$ and $Y$ is firstly introduced in [2]. $\mathcal{L}_{\gamma, U}(X, Y)$ is a meaningful extension of the family of linear operators. The authors have established the equicontinuity theorem, the uniform boundedness principle and the Banach-Steinhaus closure theorem for the extension $\mathcal{L}_{\gamma, U}(X, Y)$. Especially, for demi-linear functionals on the spaces of test functions, Ronglu Li et al have established a theory which is a natural generalization of the usual theory of distributions in their unpublished paper "Li, R, Chung, J, Kim, D: Demi-distributions, submitted".

Let $X, Y$ be topological vector spaces over the scalar field $\mathbb{K}$ and $\mathcal{N}(X)$ the family of neighborhoods of $0 \in X$. Let

$$
C(0)=\left\{\gamma \in \mathbb{K}^{\mathbb{K}}: \lim _{t \rightarrow 0} \gamma(t)=\gamma(0)=0,|\gamma(t)| \geq|t| \text { if }|t| \leq 1\right\} .
$$

Definition 1.1 [2, Definition 2.1] A mapping $f: X \rightarrow Y$ is said to be demi-linear if $f(0)$ $=0$ and there exists $\gamma \in C(0)$ and $U \in \mathcal{N}(X)$ such that every $x L X, u L U$ and $t \in\{t \in \mathbb{K}:|t| \leq 1\}$ yield $r, s \in \mathbb{K}$ for which $|r-1| \leq|\gamma(\mathrm{t})|,|s| \leq|\gamma(\mathrm{t})|$ and $f(x+t u)$ $=r f(x)+s f(u)$.

\section{Springer}

(c) $2011 \mathrm{Li}$ et al; licensee Springer. This is an Open Access article distributed under the terms of the Creative Commons Attribution License (http://creativecommons.org/licenses/by/2.0), which permits unrestricted use, distribution, and reproduction in any medium, provided the original work is properly cited. 
We denote by $\mathcal{L}_{\gamma, U}(X, Y)$ the family of demi-linear mappings related to $\gamma \in C(0)$ and $U \in \mathcal{N}(X)$, and by $\mathcal{K}_{\gamma, U}(X, Y)$ the subfamily of $\mathcal{L}_{\gamma, U}(X, Y)$ satisfying the following property: if $x L X, u L U$ and $|t| \leq 1$, then $f(x+t u)=r f(x)+s f(u)$ for some $s$ with $\mid$ $s|\leq| \gamma(\mathrm{t}) \mid$. Let

$$
X^{(\gamma, U)}=\left\{f \in \mathcal{L}_{\gamma, U}(X, \mathbb{K}): f \text { is continuous }\right\},
$$

which is called the demi-linear dual space of $X$. Obviously, $X^{\prime} \subset X^{(\gamma, u)}$.

In this article, first we discuss the spaces with non-trivial demi-linear dual, of which the usual dual may be trivial. Second we obtain a list of conclusions on the demi-linear dual pair $\left(X, X^{(\gamma, U)}\right)$. Especially, the Alaoglu-Bourbaki theorem for the pair $\left(X, X^{(\gamma, U)}\right)$ is established. We will see that many results in the usual duality theory of $\left(X, X^{\prime}\right)$ can be extended to $\left(X, X^{(\gamma, \iota)}\right)$.

Before we start, some existing conclusions about $\mathcal{L}_{\gamma, U}(X, Y)$ are given as follows. In general, $\mathcal{L}_{\gamma, U}(X, Y)$ is a large extension of $L(X, Y)$. For instance, if $\|\cdot\|: X \rightarrow[0,+\infty)$ is a norm, then $\|\cdot\| \in \mathcal{L}_{\gamma, X}(X, \mathbb{R})$ for every $\gamma \in C(0)$. Moreover, we have the following

Proposition 1.2 ([2, Theorem 2.1]) Let $X$ be a non-trivial normed space, $C>1, \delta>0$ and $U=\{u \in X:\|u\| \leq \delta\}, \gamma(t)=C t$ for $t \in \mathbb{K}$. If $Y$ is non-trivial, i.e., $Y \neq\{0\}$, then the family of nonlinear mappings in $\mathcal{L}_{\gamma, U}(X, Y)$ is uncountable, and every non-zero linear operator $T: X \rightarrow Y$ produces uncountably many of nonlinear mappings in $\mathcal{L}_{\gamma, U}(X, Y)$.

Definition 1.3 A family $\Gamma \subset Y^{X}$ is said to be equicontinuous at $x \in X$ if for every $W \in \mathcal{N}(Y)$, there exists $V \in \mathcal{N}(X)$ such that $f(x+V) \subset f(x)+W$ for all $f \in \Gamma$, and $\Gamma$ is equicontinuous on $X$ or, simply, equicontinuous if $\Gamma$ is equicontinuous at each $x \in X$.

As usual, $\Gamma \subset Y^{X}$ is said to be pointwise bounded on $X$ if $\{f(x): f \in \Gamma\}$ is bounded at each $x \in X$, and $f: X \rightarrow Y$ is said to be bounded if $f(B)$ is bounded for every bounded $B \subset X$.

The following results are substantial improvements of the equicontinuity theorem and the uniform boundedness principle in linear analysis.

Theorem 1.4 ([2, Theorem 3.1]) If $X$ is of second category and $\Gamma \subset \mathcal{L}_{\gamma, U}(X, Y)$ is a pointwise bounded family of continuous demi-linear mappings, then $\Gamma$ is equicontinuous on $X$.

Theorem 1.5 ([2, Theorem 3.3]) If $x$ is of second category and $\Gamma \subset \mathcal{L}_{\gamma, U}(X, Y)$ is a pointwise bounded family of continuous demi-linear mappings, then $\Gamma$ is uniformly bounded on each bounded subset of $X$, i.e., $\{f(x): f \in \Gamma, x \in B\}$ is bounded for each bounded $B \subset X$.

If, in addition, $X$ is metrizable, then the continuity of $f \in \Gamma$ can be replaced by boundedness of $f \in \Gamma$.

\section{Spaces with non-trivial demi-linear dual}

Lemma 2.1 Let $f \in \mathcal{L}_{\gamma, U}(X, \mathbb{K})$. For each $x \in X, u \in U$ and $|t| \leq 1$, we have

$$
\begin{aligned}
& |f(t u)| \leq|\gamma(t)||f(u)| \\
& |f(x+t u)-f(x)| \leq|\gamma(t)|(|f(x)|+|f(u)|) .
\end{aligned}
$$


Proof. Since $f \in \mathcal{L}_{\gamma, U}(X, \mathbb{K})$, for each $x \in X, u \in U$ and $|t| \leq 1$, we have $f(x+t u)=r f$ $(x)+s f(u)$ where $|r-1| \leq|\gamma(t)|$ and $|s| \leq|\gamma(t)|$. Then

$$
|f(x+t u)-f(x)|=|(r-1) f(x)+s f(u)| \leq|r-1||f(x)|+|s||f(u)| \leq|\gamma(t)|(|f(x)|+|f(u)|),
$$

which implies (2). Then (1) holds by letting $x=0$ in (2).

Theorem 2.2 Let $X$ be a topological vector space and $f: X \rightarrow[0,+\infty)$ a function satisfying

$(*) f(0)=0, f(-x)=f(x)$ and $f(x+y) \leq f(x)+f(y)$ whenever $x, y \in X$.

Then, for every $\gamma \in C(0)$ and $U \in \mathcal{N}(X)$, the following (I), (II), and (III) are equivalent:

(I) $f \in \mathcal{L}_{\gamma, U}(X, \mathbb{R})$;

(II) $f(t u) \leq|\gamma(t)| f(u)$ whenever $u \in U$ and $|t| \leq 1$;

(III) $f \in \mathcal{K}_{\gamma, U}(X, \mathbb{R})$.

Proof. (I) $\Rightarrow$ (II). By Lemma 2.1 .

(II) $\Rightarrow$ (III). Let $x \in X, u \in U$ and $|t| \leq 1$. Then

$$
f(x)-|\gamma(t)| f(u) \leq f(x)-f(t u) \leq f(x+t u) \leq f(x)+f(t u) \leq f(x)+|\gamma(t)| f(u) .
$$

Define $\phi:[-|\gamma(t)|,|\gamma(t)|] \rightarrow \mathbb{R}$ by $\phi(\alpha)=f(x)+\alpha f(u)$. Then $\phi$ is continuous and

$$
\varphi(-|\gamma(t)|)=f(x)-|\gamma(t)| f(u) \leq f(x+t u) \leq f(x)+|\gamma(t)| f(u)=\varphi(|\gamma(t)|) .
$$

So there is $s \in[-|\gamma(t)|,|\gamma(t)|]$ such that $f(x+t u)=\gamma(s)=f(x)+s f(u)$.

(III) $\Rightarrow(\mathrm{I}) . \mathcal{K}_{\gamma, U}(X, \mathbb{R}) \subset \mathcal{L}_{\gamma, U}(X, \mathbb{R})$.

In the following Theorem 2.2, we want to know whether a paranorm on a topological vector space $X$ is in $\mathcal{K}_{\gamma, U}(X, \mathbb{R})$ for some $\gamma$ and $U$. However, the following example shows that this is invalid.

Example 2.3 Let $\omega$ be the space of all sequences with the paranorm $\|\cdot\|$ :

$$
\|x\|=\sum_{j=1}^{\infty} \frac{1}{2^{j}} \frac{\left|x_{j}\right|}{1+\left|x_{j}\right|}, \forall x=\left(x_{j}\right) \in \omega .
$$

Then, for every $\gamma \in C(0)$ and $U_{\varepsilon}=\left\{u=\left(u_{j}\right):\|u\|<\varepsilon\right\}$, we have $\|\cdot\| \notin \mathcal{L}_{\gamma, U}(\omega, \mathbb{R})$. Otherwise, there exists $\gamma \in C(0)$ and $\varepsilon>0$ such that $\|\cdot\| \notin \mathcal{L}_{\gamma, U}(\omega, \mathbb{R})$ and hence

$$
\left\|\frac{1}{n} u\right\| \leq\left|\gamma\left(\frac{1}{n}\right)\right|\|u\|, \text { for all } u \in U_{\varepsilon} \text { and } n \in \mathbb{N}
$$

by Theorem 2.2. Pick $N \in \mathbb{N}$ with $\frac{1}{2^{N}}<\varepsilon$. Let $u_{n}=(0, \cdots, 0, \stackrel{(N)}{n}, 0, \cdots), \forall n \in \mathbb{N}$.

Then $\left\|u_{n}\right\|=\frac{1}{2^{N}} \frac{n}{1+n}<\frac{1}{2^{N}}<\varepsilon$ implies $u_{n} \in U_{\varepsilon}$ for each $N \in \mathbb{N}$. It follows from

$$
\left|\gamma\left(\frac{1}{n}\right)\right| \geq \frac{\left\|\frac{1}{n} u_{n}\right\|}{\left\|u_{n}\right\|}=\left(\frac{1}{2^{N}} \frac{1}{1+1}\right) /\left(\frac{1}{2^{N}} \frac{n}{1+n}\right)=\frac{1}{2} \frac{1+n}{n}>\frac{1}{2}, \forall n \in \mathbb{N},
$$

that $\gamma\left(\frac{1}{n}\right) \nrightarrow 0$ as $n \rightarrow \infty$, which contradicts $\gamma \in C(0)$. 
Note that the space $\omega$ in Example 2.3 has a Schauder basis. The following corollary shows that the set of nonlinear demi-linear continuous functionals on a Hausdorff topological vector space with a Schauder basis has an uncountable cardinality.

Corollary 2.4 Let $X$ be a Hausdorff topological vector space with a Schauder basis.

Then for every $\gamma \in C(0)$ and $U \in \mathcal{N}(X)$, the demi-linear dual $X^{(\gamma, U)}=\left\{f \in \mathcal{L}_{\gamma, U}(X, \mathbb{R}): f\right.$ is continuous $\}$ is uncountable.

Proof. Let $\left\{b_{k}\right\}$ be a Schauder basis of $X$. There is a family $P$ of non-zero paranorms on $X$ such that the vector topology on $X$ is just $\sigma P$, i.e., $x_{\alpha} \rightarrow x$ in $X$ if and only if $\| x_{\alpha}$ $-x \| \rightarrow 0$ for each $\|\cdot\| \in P([[1]$, p.55]).

Pick $\|\cdot\| \in P$. Then $\left\|\sum_{k=1}^{\infty} s_{k} b_{k}\right\| \neq 0$ for some $\sum_{k=1}^{\infty} s_{k} b_{k} \in X$ and hence $\left\|s_{k_{0}} b_{k_{0}}\right\| \neq 0$ for some $k_{0} \in \mathbb{N}$. For non-zero $c \in \mathbb{K}$, define $f_{c}: X \rightarrow[0,+\infty)$ by

$$
f_{c}\left(\sum_{k=1}^{\infty} r_{k} b_{k}\right)=\left|c r_{k_{0}}\right||| s_{k_{0}} b_{k_{0}} \|
$$

Obviously, $f_{c}$ is continuous and satisfies the condition (*) in Theorem 2.2. Let $\gamma \in C$ (0), $\sum_{k=1}^{\infty} r_{k} b_{k} \in X$ and $|t| \leq 1$. Then

$$
f_{c}\left(t \sum_{k=1}^{\infty} r_{k} b_{k}\right)=\left|c t r_{k_{0}}\right||| s_{k_{0}} b_{k_{0}}\left\|=|t|\left|c r_{k_{0}}\right||| s_{k_{0}} b_{k_{0}}\right\|=|t| f_{c}\left(\sum_{k=1}^{\infty} r_{k} b_{k}\right) \leq|\gamma(t)| f_{c}\left(\sum_{k=1}^{\infty} r_{k} b_{k}\right)
$$

and hence $f_{c} \in \mathcal{K}_{\gamma, U}(X, \mathbb{R}) \subset \mathcal{L}_{\gamma, U}(X, \mathbb{R})$ for all $U \in \mathcal{N}(X)$ by Theorem 2.2. Thus, $\left\{f_{c}: 0 \neq c \in \mathbb{K}\right\} \subset X^{(\gamma, U)}$ for all $\gamma \in C(0)$ and $U \in \mathcal{N}(X)$.

Example 2.5 As in Example 2.3, the space $(\omega,\|\cdot\|)$ is a Hausdorff topological vector space with the Schauder base $\left\{e_{n}=(0, \cdots, 0, \stackrel{(n)}{1}, 0, \cdots): n \in \mathbb{N}\right\}$. Define $f_{c, n}: \omega \rightarrow \mathbb{R}$ with $f_{c, n}(u)=\left|c u_{n}\right|$ where $u=\left(u_{j}\right) \in \omega$. Then we have

$$
\left\{f_{c, n}: 0 \neq c \in \mathbb{K}, n \in \mathbb{N}\right\} \subset \omega^{(\gamma, U)}=\left\{f \in \mathcal{L}_{\gamma, U}(\omega, \mathbb{R}): f \text { is continuous }\right\}
$$

for every $\gamma \in C(0)$ and $U \in \mathcal{N}(\omega)$ by Corollary 2.4.

Recall that a $p$-seminorm $\|\cdot\|(0<p \leq 1)$ on a vector space $E$ is characterized by $\|x\|$ $\geq 0,\|t x\|=|t|^{p}|| x||$ and $\|x+y\| \leq\|x\|+\|y\|$ for all $t \in \mathbb{K}$ and $x, y \in E$. If, in addition, $\|x\|=0$ implies $x=0$, then, $\|\cdot\|$ is called a $p$-norm on $E$.

Definition 2.6 ([[3], p. 11][[4], Sec. 2]) A topological vector space $X$ is semiconvex if and only if there is a family $\left\{p_{\alpha}\right\}$ of (continuous) $k_{\alpha}$-seminorms $\left(0<k_{\alpha} \leq 1\right)$ such that the sets $\left\{x \in X: p_{\alpha}(x)<1\right\}$ form a neighborhood basis at 0 , that is,

$$
\left\{\left\{x: p_{\alpha}(x)<\frac{1}{n}\right\}: p_{\alpha} \in P, n \in N\right\}
$$

is a base of $\mathcal{N}(X)$, where $P$ is the family of all continuous $p$-seminorms with $0<p \leq 1$.

A topological vector space $X$ is locally bounded if and only if its topology is given by a $p$-norm $(0<p \leq 1)([[5], \$ 15$, Sec. 10]).

Clearly, locally bounded spaces and locally convex spaces are both semiconvex. 
Corollary 2.7 Let $X$ be a semiconvex Hausdorff topological vector space and $p_{0}$ a continuous $k_{0}$-seminorm $\left(0<k_{0} \leq 1\right)$ on $X$. Then for $U_{0}=\left\{x \in X: p_{0}(x) \leq 1\right\} \in \mathcal{N}(X)$ and $\gamma(\cdot)=e|\cdot|^{k_{0}} \in \mathbb{K}^{\mathbb{K}}$, the demi-linear dual

$$
X^{\left(\gamma, U_{0}\right)}=\left\{f \in \mathcal{L}_{\gamma, U_{0}}(X, \mathbb{R}): f \text { is continuous }\right\}
$$

is uncountable. Especially, $\left\{p_{0}(\cdot), \sin \left(p_{0}(\cdot)\right), e^{p_{0}(\cdot)}-1\right\} \subset X^{\left(\gamma, U_{0}\right)}$.

Proof. Let $P$ be the family of all continuous $k_{\alpha}$-seminorms with $0<k_{\alpha} \leq 1$. Obviously, the functionals in $P$ satisfy the condition (*) in Theorem 2.2. Moreover, for each $p_{\alpha} \in$ $P$ with $k_{\alpha} \geq k_{0}$, we have

$$
c p_{\alpha}(t x)=c|t|^{k_{\alpha}} p_{\alpha}(x) \leq c|t|^{k_{0}} p_{\alpha}(x) \leq|\gamma(t)| c p_{\alpha}(x), \text { for all } x \in X,|t| \leq 1 \text { and } c \in \mathbb{K},
$$

and hence $\left\{c p_{\alpha}: c \in \mathbb{K}, k_{\alpha} \geq k_{0}\right\} \subset X^{\left(\gamma, U_{0}\right)}$ by Theorem 2.2.

Define $f: X \rightarrow \mathbb{R}$ by $f(x)=\sin \left(p_{0}(x)\right), \forall x \in X$. For each $x \in X, u \in U_{0}$ and $|t| \leq 1$, there exists $s \in\left[-|t|^{k_{0}},|t|^{k_{0}}\right]$ and $\theta \in[0,1]$ such that

$$
\sin \left(p_{0}(x+t u)\right)=\sin \left(p_{0}(x)+s p_{0}(u)\right)=\sin \left(p_{0}(x)\right)+\cos \left(p_{0}(x)+\theta s p_{0}(u)\right) s p_{0}(u),
$$

i.e.,

$$
f(x+t u)=f(x)+\cos \left(p_{0}(x)+\theta s p_{0}(u)\right) \frac{p_{0}(u)}{\sin \left(p_{0}(u)\right)} s f(u),
$$

where

$$
\left|\cos \left(p_{0}(x)+\theta s p_{0}(u)\right) \frac{p_{0}(u)}{\sin \left(p_{0}(u)\right)} s\right| \leq \frac{\pi}{2}|t|^{k_{0}} \leq e|t|^{k_{0}}=|\gamma(t)|,
$$

which implies that $f(\cdot)=\sin \left(p_{0}(\cdot)\right) \in X^{\left(\gamma, U_{0}\right)}$.

Define $g: X \rightarrow \mathbb{R}$ by $g(x)=e^{p_{0}(x)}-1, \forall x \in X$. For each $x \in X, u \in U_{0}$ and $|t| \leq 1$, there exists $s \in\left[-|t|^{k_{0}},|t|^{k_{0}}\right]$ such that

$$
e^{p_{0}(x+t u)}-1=e^{p_{0}(x)+s p_{0}(u)}-1=e^{s p_{0}(u)}\left(e^{p_{0}(x)}-1\right)+\frac{e^{s p_{0}(u)}-1}{e^{p_{0}(x)}-1}\left(e^{p_{0}(x)}-1\right),
$$

i.e.,

$$
g(x+t u)=e^{s p_{0}(u)} g(x)+\frac{e^{s p_{0}(u)}-1}{e^{p_{0}(x)}-1} g(u) .
$$

Then, there exists $\theta, \eta \in[0,1]$ for which

$$
\left|e^{s p_{0}(u)}-1\right|=\left|e^{\theta s p_{0}(u)} s p_{0}(u)\right| \leq e|s| \leq e|t|^{k_{0}}=|\gamma(t)|
$$

and

$$
\left|\frac{e^{s p_{0}(u)}-1}{e^{p_{0}(x)}-1}\right|=\left|\frac{e^{\theta s p_{0}(u)} s p_{0}(u)}{e^{\eta p_{0}(u)} p_{0}(u)}\right| \leq e^{\theta s p_{0}(u)}|s| \leq e|s| \leq e|t|^{k_{0}}=|\gamma(t)| .
$$

Thus, $g(\cdot)=e^{p_{0}(\cdot)}-1 \in X^{\left(\gamma, U_{0}\right)}$. 
Example 2.8 For $0<p<1$, let $L^{p}(0,1)$ be the space of equivalence classes of measurable functions on $[0,1]$, with

$$
\|f\|=\int_{0}^{1}|f(t)|^{p} d t<\infty .
$$

Then $\left(L^{p}(0,1),\|\cdot\|\right)^{\prime}=\{0\}([[1], p .25])$. However, $L^{p}(0,1)$ is locally bounded and hence semiconvex. By Corollary 2.7, if $U_{0}=\{f:|| f|| \leq 1\}$ and $\gamma(\cdot)=e|\cdot|^{p} \in C(0)$, then the demi-linear dual $\left(L^{p}(0,1),\|\cdot\|\right)^{\left(\gamma, U_{0}\right)}$ contains various non-zero functionals.

A conjecture is that each topological vector space has a nontrivial demi-linear dual space. However, this is invalid, even for separable Fréchet space.

Example 2.9 Let $\mathcal{M}(0,1)$ be the space of equivalence classes of measurable functions on $[0,1]$, with

$$
\|f\|=\int_{0}^{1} \frac{|f(t)|}{1+|f(t)|} d t .
$$

Then $\mathcal{M}(0,1)$ is a separable Fréchet space with trivial dual. In fact, the demi-linear dual space of $\mathcal{M}(0,1)$ is also trivial, that is,

$$
(\mathcal{M}(0,1),\|\cdot\|)^{(\gamma, U)}=\{0\} \text { for each } \gamma \in C(0) \text { and } U \in \mathcal{N}(\mathcal{M}(0,1))
$$

Let $u \in(\mathcal{M}(0,1),\|\cdot\|)^{(\gamma, U)}$. Let $N \in \mathbb{N}$ be such that $\left\|f_{k}\right\| \leq \frac{1}{N}$ implies $f \in U$ and $\mid u$ $(f) \mid<1$. Given $f \in \mathcal{M}(0,1)$, write $f=\sum_{k=1}^{N} f_{k}$ where $f_{k}=0$ off $\left[\frac{k-1}{N}, \frac{k}{N}\right]$. Then

$$
\begin{aligned}
& u(f)=u\left(\sum_{k=1}^{N} f_{k}\right)=u\left(\sum_{k=1}^{N-1} f_{k}+f_{N}\right) \\
& =r_{N} u\left(\sum_{k=1}^{N-1} f_{k}\right)+s_{N} u\left(f_{N}\right) \\
& =r_{N} r_{N-1} u\left(\sum_{k=1}^{N-2} f_{k}\right)+r_{N} s_{N-1} u\left(f_{N-1}\right)+s_{N} u\left(f_{N}\right)^{\text {so }} \\
& =\cdots \\
& =r_{N} \cdots r_{3} r_{2} u\left(f_{1}\right)+r_{N} \cdots r_{3} s_{2} u\left(f_{2}\right)+\cdots \\
& +r_{N} s_{N-1} u\left(f_{N-1}\right)+s_{N} u\left(f_{N}\right) \text {, } \\
& u(f)=u\left(\sum_{k=1}^{N} f_{k}\right)=u\left(\sum_{k=1}^{N-1} f_{k}+f_{N}\right) \\
& =r_{N} u\left(\sum_{k=1}^{N-1} f_{k}\right)+s_{N} u\left(f_{N}\right) \\
& =r_{N} r_{N-1} u\left(\sum_{k=1}^{N-2} f_{k}\right)+r_{N} s_{N-1} u\left(f_{N-1}\right)+s_{N} u\left(f_{N}\right) \\
& =\cdots \\
& =r_{N} \cdots r_{3} r_{2} u\left(f_{1}\right)+r_{N} \cdots r_{3} s_{2} u\left(f_{2}\right)+\cdots \\
& +r_{N} s_{N-1} u\left(f_{N-1}\right)+s_{N} u\left(f_{N}\right) \text {, }
\end{aligned}
$$


where $\left|r_{i}-1\right| \leq|\chi(1)|$ and $\left|s_{i}\right| \leq|\chi(1)|$ for $2 \leq I \leq N$. Then

$$
\begin{aligned}
& |u(f)| \leq(1+|\gamma(1)|)^{N-1}\left|u\left(f_{1}\right)\right|+(1+|\gamma(1)|)^{N-2}|\gamma(1)|\left|u\left(f_{2}\right)\right|+\cdots \\
& +(1+|\gamma(1)|)|\gamma(1)|\left|u\left(f_{N-1}\right)\right|+|\gamma(1)|\left|u\left(f_{N}\right)\right| \\
& \leq(1+|\gamma(1)|)^{N-1}+(1+|\gamma(1)|)^{N-2}|\gamma(1)|+\cdots \\
& +(1+|\gamma(1)|)|\gamma(1)|+|\gamma(1)| \\
& =2(1+|\gamma(1)|)^{N-1}-1 \text {. }
\end{aligned}
$$

So $\sup _{f \in \mathcal{M}(0,1)}|u(f)|<+\infty$. Since $\left\|n f_{k}\right\| \leq \frac{1}{N}$ for each $n \in \mathbb{N}$ and $1 \leq k \leq N$, we have $\left\{n f_{k}: n \in \mathbb{N}, k \in \mathbb{N}\right\} \subset U$. Then by Lemma 2.1,

$$
\left|u\left(f_{k}\right)\right|=\left|u\left(\frac{1}{n}\left(n f_{k}\right)\right)\right| \leq\left|\gamma\left(\frac{1}{n}\right)\right|\left|u\left(n f_{k}\right)\right| \leq\left|\gamma\left(\frac{1}{n}\right)\right| \sup _{f \in \mathcal{M}(0,1)}|u(f)|
$$

holds for all $n \in \mathbb{N}$ and $1 \leq k \leq N$. Letting $n \rightarrow \infty$, (7) implies $u\left(f_{k}\right)=0$ for $1 \leq k \leq N$. Hence, $|u(f)|=0$ by (4). Thus, $u=0$.

\section{Conclusions on the demi-linear dual pair $\left(X, X^{(\gamma, U)}\right)$}

Henceforth, $X$ and $Y$ are topological vector spaces over $\mathbb{K}, \mathcal{N}(X)$ is the family of neighborhoods of $0 \in X$, and $X^{(\gamma, L)}$ is the family of continuous demi-linear functionals in $\mathcal{L}_{\gamma, U}(X, \mathbb{K})$. Recall that for usual dual pair $\left(X, X^{\prime}\right)$ and $A \subset X$, the polar of $A$, written as $A^{\circ}$, is given by

$$
A^{\circ}=\left\{f \in X^{\prime}:|f(x)| \leq 1, \forall x \in A\right\} .
$$

In this article, for the demi-linear dual pair $\left(X, X^{(\gamma, L)}\right)$ and $A \subset X$, we denote the polar of $A$ by $A^{*}$, which is given by

$$
A^{\bullet}=\left\{f \in X^{(\gamma, U)}:|f(x)| \leq 1, \forall x \in A\right\} .
$$

Similarly, for $S \subset X^{(\gamma, U)}$,

$$
S^{\bullet}=\{x \in X:|f(x)| \leq 1, \forall f \in S\} .
$$

Lemma 3.1. Let $f \in \mathcal{L}_{\gamma, U}(X, Y)$. For every $u \in U$ and $n \in \mathbb{N}$,

$$
f(n u)=\alpha f(u), \text { where }|\alpha| \leq 2(1+|\gamma(1)|)^{n-1}-1 .
$$

Proof. It is similar to the proof of (3)-(6) in Example 2.9.

Lemma 3.2. Let $S \subset X^{(\gamma, L)}$. If $S$ is equicontinuous at $0 \in X$, then, $S^{\bullet} \in \mathcal{N}(X)$ and sup $f \in S, x \in B|f(x)|<+\infty$ for every bounded $B \subset X$.

Proof. Assume that $S$ is equicontinuous at $0 \in X$. There is $U \in \mathcal{N}(X)$ such that $|f(x)|$ $<1$ for all $f \in S$ and $x \in V$. Then $V \subset S^{\bullet}$ and hence $S^{\bullet} \in \mathcal{N}(X)$.

Let $B \subset X$ be bounded. Since $S^{\bullet} \cap U \in \mathcal{N}(X)$, we have $\frac{1}{m} B \subset S^{\bullet} \cap U$ for some $m \in$ $\mathbb{N}$. Then for each $f \in S$ and $x \in B$,

$$
|f(x)|=\left|f\left(m \frac{x}{m}\right)\right|=|\alpha|\left|f\left(\frac{x}{m}\right)\right| \leq|\alpha| \leq 2(1+|\gamma(1)|)^{m-1}-1
$$


by Lemma 3.1. Hence, $\sup _{f \in S, x \in B}|f(x)| \leq 2(1+|\gamma(1)|)^{m-1}-1<+\infty$.

Lemma 3.3. Let $S \subset X^{(\gamma, U)}$. Then $S$ is equicontinuous on $X$ if and only if $S$ is equicontinuous at $0 \in X$.

Proof. Assume that $S$ is equicontinuous at $0 \in X$. There is $W \in \mathcal{N}(X)$ such that $\mid f$ $(\omega) \mid<1$ for all $f \in S$ and $\omega \in W$.

Let $x \in X$ and $\varepsilon>0$. By Lemma 3.2, sup $f \in S|f(x)|=M<+\infty$. Observing $\lim _{t \rightarrow o} \gamma(t)$ $=0$, pick $\delta \in(0,1)$ such that $\left|\gamma\left(\frac{\delta}{2}\right)\right|<\frac{\varepsilon}{2(M+1)}$. By Lemma 2.1, for $f \in S$ and $u=\frac{\delta}{2} u_{0} \in \frac{\delta}{2}(W \cap U)$, we have

$$
|f(x+u)-f(x)|=\left|f\left(x+\frac{\delta}{2} u_{0}\right)-f(x)\right| \leq\left|\gamma\left(\frac{\delta}{2}\right)\right|\left(|f(x)|+\left|f\left(u_{0}\right)\right|\right)<\frac{\varepsilon}{2(M+1)}(M+1)<\varepsilon .
$$

Thus, $f\left[x+\frac{\delta}{2}(W+U)\right] \subset f(x)+\{z \in \mathbb{K}:|z|<\varepsilon\}$ for all $f \in S$, i.e., $S$ is equicontinuous at $x$.

Theorem 3.4. Let $S \subset X^{(\gamma, U)}$. Then $S$ is equicontinuous on $X$ if and only if $S^{\bullet} \in \mathcal{N}(X)$. Proof. If $S$ is equicontinuous, then $S^{\bullet} \in \mathcal{N}(X)$ by Lemma 3.2.

Assume that $S^{\bullet} \in \mathcal{N}(X)$ and $\varepsilon>0$. Since $\lim _{t \rightarrow 0} \gamma(t)=\gamma(0)=0$, there is $\delta>0$ such that $|\gamma(t)|<\varepsilon$ whenever $|t|<\delta$. For $f \in S$ and $x=\frac{\delta}{2} x_{0} \in \frac{\delta}{2}\left(S^{\bullet} \cap U\right)$, we have $\left|f\left(x_{0}\right)\right| \leq 1$ and $\quad|f(x)|=\left|f\left(\frac{\delta}{2} x_{0}\right)\right| \leq\left|\gamma\left(\frac{\delta}{2}\right)\right|\left|f\left(x_{0}\right)\right|<\varepsilon \quad$ by Lemma 2.1. Thus, $f\left[\frac{\delta}{2}\left(S^{\bullet} \cap U\right)\right] \subset\{z \in \mathbb{K}:|z|<\varepsilon\}$ for all $f \in S$, i.e., $S$ is equicontinuous at $0 \in X$. By Lemma 3.3, $S$ is equicontinuous on $X$.

The following simple fact should be helpful for further discussions.

Example 3.5. Let $\left(L^{p}(0,1),\|\cdot\|\right)$ be as in Example 2.8, $U=\{f:\|f\| \leq 1\}$ and $\gamma(t)=e$ $|t|^{p}$ for $t \in \mathbb{K}$. Then $\left(L^{p}(0,1),\|\cdot\|\right)^{(\gamma, U)}$ contains non-zero continuous functionals such as $\|\cdot\|$, sin $\|\cdot\|$, e $e^{\|\cdot\|}-1$, etc. Since $(\alpha f)(\cdot)=\alpha f(\cdot)$ for $\alpha \in \mathbb{K}$ and $f \in\left(L^{p}(0,1),\|\cdot\|\right)^{(\gamma, U)}$, it follows from $e^{\|\cdot\|}-1 \in\left(L^{p}(0,1),\|\cdot\|\right)^{(\gamma, U)}$ that $\frac{1}{e}\left(e^{\|\cdot\|}-1\right) \in\left(L^{p}(0,1),\|\cdot\|\right)^{(\gamma, U)}$. If $u \in$ $U$, then $\|u\| \leq 1, \mid \sin \|u\|\|\leq\| u \| \leq 1$ and $\left|\frac{1}{e}\left(e^{\|u\|}-1\right)\right| \leq \frac{e-1}{e}<1$. Thus, if $V$ is a neighborhood of $0 \in L^{p}(0,1)$ such that $V \subset U$, then $V$ contains non-zero functionals such as $\|\cdot\|$, sin $\|\cdot\|, \frac{1}{e}\left(e^{\|\cdot\|}-1\right)$, etc.

Corollary 3.6. For every $U, V \in \mathcal{N}(X)$ and $\gamma \in C(0), V=\left\{f \in X^{(\gamma, U)}:|f(x)| \leq 1, \forall x \in\right.$ $V\}$ is equicontinuous on $X$.

Proof. Let $x \in V$. Then $|f(x)| \leq 1, \forall f \in V^{*}$, i.e., $x \in\left(V^{*}\right)^{\bullet}$. Thus, $V \subset\left(V^{*}\right)^{\bullet}$ and so $\left(V^{\bullet}\right)^{\bullet} \in \mathcal{N}(X)$. By Theorem 3.4, $V$ is equicontinuous on $X$.

Corollary 3.7. If $X$ is of second category and $S \subset X^{(\gamma, U)}$ is pointwise bounded on $X$, then $S^{\bullet} \in \mathcal{N}(X)$.

Proof. By Theorem 1.4, $S$ is equicontinuous on $X$. Then $S^{\bullet} \in \mathcal{N}(X)$ by Theorem 3.4.

Corollary 3.8. Let $X$ be a semiconvex space and $S \subset X^{(\gamma, U)}$. Then $S$ is equicontinuous on $x$ if and only if there exist finitely many continuous $k_{i}$-seminorm $p_{i}$ 's $\left(0<k_{i} \leq 1,1 \leq i\right.$ $\leq n<+\infty$ ) on $x$ such that

$$
\sup _{f \in S} \sup _{p_{i}(x) \leq 1,1 \leq i \leq n}|f(x)|<+\infty .
$$

In particular, for a p-seminormed space $(X,\|\cdot\|)(\|\cdot\|$ is a $p$-seminorm for some $p \in$ $(0,1]$, especially, a norm when $p=1)$ and $S \subset X^{(\gamma, U)}, S$ is equicontinuous on $x$ if and 
only if

$$
\sup _{f \in S} \sup _{\|x\| \leq 1}|f(x)|<+\infty \text {. }
$$

Proof. Assume that $S$ is equicontinuous. Then $S^{\bullet} \in \mathcal{N}(X)$ by Theorem 3.4. According to Definition 2.6 , there exist finitely many continuous $k_{i}$-seminorm $p_{i}$ 's $\left(0<k_{i} \leq 1\right.$, $1 \leq i \leq n<+\infty)$ and $\varepsilon>0$ such that

$$
\left\{x \in X: p_{i}(x)<\varepsilon, 1 \leq i \leq n\right\} \subset S^{\bullet} \cap U .
$$

Let $f \in S$ and $p_{i}(x) \leq 1,1 \leq i \leq n$. Pick $n_{0} \in \mathbb{N}$ for which $\left(\frac{1}{n_{0}}\right)^{k_{0}}<\varepsilon$, where $k_{0}=$ $\min _{1 \leq i \leq n} k_{i}$. Then

$$
p_{i}\left(\frac{x}{n_{0}}\right)=\left(\frac{1}{n_{0}}\right)^{k_{i}} p_{i}(x) \leq\left(\frac{1}{n_{0}}\right)^{k_{0}} p_{i}(x)<\varepsilon, \text { for } 1 \leq i \leq n,
$$

which implies $\frac{x}{n_{0}} \in S^{\bullet} \cap U$ and hence $\left|f\left(\frac{x}{n_{0}}\right)\right| \leq 1$. By Lemma 3.1,

$$
|f(x)|=\left|f\left(n_{0} \frac{x}{n_{0}}\right)\right|=\left|\alpha f\left(\frac{x}{n_{0}}\right)\right| \leq|\alpha| \leq 2(1+|\gamma(1)|)^{n_{0}-1}-1
$$

Thus, $\sup _{f \in S} \sup _{p_{i}(x) \leq 1,1 \leq i \leq n}|f(x)| \leq 2(1+|\gamma(1)|)^{n_{0}-1}-1<+\infty$.

Conversely, suppose that $p_{i}$ is a continuous $k_{i}$-seminorm with $0<k_{i} \leq 1$ for $1 \leq i \leq n$ $<+\infty$, and (8) holds. Let $A=\left\{\frac{1}{M+1} f: f \in S\right\}$. Then $A \subset X^{(\gamma, U)}$ and

$$
\sup _{g \in A} \sup _{p_{i}(x) \leq 1,1 \leq i \leq n}|g(x)|=\frac{1}{1+M} \sup _{f \in S} \sup _{p_{i}(x) \leq 1,1 \leq i \leq n}|f(x)|=\frac{M}{1+M}<1,
$$

i.e., $\left\{x \in X: p_{i}(x) \leq 1,1 \leq i \leq n\right\} \subset A^{\bullet}$ and so $A^{\bullet} \in \mathcal{N}(X)$. By Theorem 3.4, $A^{\bullet}$ is equicontinuous on $X$ and $S=(1+M) A$ is also equicontinuous on $X$.

Lemma 3.9. Let $C(X, \mathbb{K})=\left\{f \in \mathbb{K}^{X}: f\right.$ is continuous $\}$. For $S \subset C(X, \mathbb{K})$, the following (I) and (II) are equivalent.

(I) $S$ is equicontinuous on $X$.

(II) If $\left(x_{\alpha}\right)_{\alpha \in} I$ is a net in $x$ such that $x_{\alpha} \rightarrow x \in X$, then $\lim _{\alpha} f\left(x_{\alpha}\right)=f(x)$ uniformly for $f \in S$.

Proof. (I) $\Rightarrow$ (II). Let $\varepsilon>0$ and $x_{\alpha} \rightarrow x$ in $X$. Since $S$ is equicontinuous on $X$, there is $W \in \mathcal{N}(X)$ such that

$$
|f(x+w)-f(x)|<\varepsilon, \quad \text { for all } f \in S \text { and } w \in W .
$$

Since $x_{\alpha} \rightarrow x$, there is an index $\alpha_{0}$ such that $x_{\alpha}-x \in W$ for all $\alpha \geq \alpha_{0}$. Then

$$
\left|f\left(x_{\alpha}\right)-f(x)\right|=\left|f\left(x+x_{\alpha}-x\right)-f(x)\right|<\varepsilon, \text { for all } f \in S \text { and } \alpha>\alpha_{0} .
$$

Thus, $\lim _{\alpha} f\left(x_{\alpha}\right)=f(x)$ uniformly for $f \in S$.

$(\mathrm{II}) \Rightarrow(\mathrm{I})$. Suppose that (II) holds but there exists $x \in X$ such that $S$ is not equicontinuous at $x$.

Then there exists $\varepsilon>0$ such that for every $V \in \mathcal{N}(X)$, we can choose $f_{v} \in S$ and $z_{v}$ $\in V$ for which 


$$
\left|f_{v}\left(x+z_{v}\right)-f_{v}(x)\right| \geq \varepsilon
$$

Since $(\mathcal{N}(X), \supset)$ is a directed set, we have $\left(x+z_{v}\right)_{V \in \mathcal{N}(X)}$ is a net in $X$. For every $x+z_{v} \in x+V \subset x+W$ for all $V \in \mathcal{N}(X)$ with $W \supset V$,

$$
x+z_{v} \in x+V \subset x+W \text { for all } V \in \mathcal{N}(X) \text { with } W \supset V,
$$

that is, $\lim _{v}\left(x+z_{v}\right)=x$.

By (II), there exists $W_{0} \in \mathcal{N}(X)$ such that $\left|f\left(x+z_{v}\right)-f(x)\right|<\varepsilon$ for all $f \in S$ and $V \in \mathcal{N}(X)$ with $W_{0} \supset V$. Then $\left|f_{v}\left(x+z_{v}\right)-f_{v}(x)\right|<\varepsilon$ for all $V \in \mathcal{N}(X)$ with $W_{0} \supset V$. This contradicts (9) established above. Therefore, (II) implies (I).

We also need the following generalization of the useful lemma on interchange of limit operations due to E. H. Moore, whose proof is similar to the proof of Moore lemma ([[6], p. 28]).

Lemma 3.10. Let $D_{1}$ and $D_{2}$ be directed sets, and suppose that $D_{1} \times D_{2}$ is directed by the relation $\left(d_{1}, d_{2}\right) \leq\left(d_{1}^{\prime}, d_{2}^{\prime}\right)$, which is defined by $d_{1} \leq d_{1}^{\prime}$ and $d_{2} \leq d_{2}^{\prime}$. Let $f: D_{1} \times$ $D_{2} \rightarrow X$ be a net in the complete topological vector space X. Suppose that:

(a) for each $d_{2} \in D_{2}$, the limit $g\left(d_{2}\right)=\lim _{D_{1}} f\left(d_{1}, d_{2}\right)$ exists, and

(b) the limit $h\left(d_{1}\right)=\lim _{D_{2}} f\left(d_{1}, d_{2}\right)$ exists uniformly on $D_{1}$.

Then, the three limits

$$
\lim _{D_{2}} g\left(d_{2}\right), \lim _{D_{1}} h\left(d_{1}\right), \lim _{D_{1} \times D_{2}} f\left(d_{1}, d_{2}\right)
$$

all exist and are equal.

We now establish the Alaoglu-Bourbaki theorem ([[1], p. 130]) for the pair $\left(X, X^{(\gamma, L)}\right)$, where $X$ is an arbitrary non-trivial topological vector space.

Let $\mathbb{K}^{X}$ be the family of all scalar functions on $X$. With the pointwise operations $(f+$ $g)(x)=f(x)+g(x)$ and $(t f)(x)=t f(x)$ for $x \in X$ and $t \in \mathbb{K}$, we have $x: \mathbb{K}^{X} \rightarrow \mathbb{K}$ is a linear space and each $x \in X$ defines a linear functional $x: \mathbb{K}^{X} \rightarrow \mathbb{K}$ by letting $x(f)=f(x)$ for $f \in \mathbb{K}^{X}$. In fact, for $f, g \in \mathbb{K}^{X}$ and $\alpha, \beta \in \mathbb{K}$,

$$
x(\alpha f+\beta g)=(\alpha f+\beta g)(x)=\alpha x(f)+\beta x(g) .
$$

Then, each $x \in X$ produces a vector topology $\omega x$ on $\mathbb{K}^{X}$ such that

$$
f_{\alpha} \rightarrow f \operatorname{in}\left(\mathbb{K}^{X}, \omega x\right) \text { if and only if } f_{\alpha}(x) \rightarrow f(x)([1, \text { p.12, p.38] }) .
$$

The vector topology $\mathbf{V}\{\omega x: x \in X\}$ is just the weak * topology in the pair $\left(X, \mathbb{K}^{X}\right)$, and $f_{\alpha} \rightarrow f$ in $\left(\mathbb{K}^{X}\right.$, weak $\left.*\right)$ if and only if $f_{\alpha}(x) \rightarrow f(x)$ for each $x \in X([[1], \mathrm{p} .12, \mathrm{p}$. $38])$. Note that weak* is a Hausdorff locally convex topology on $\mathbb{K}^{X}$.

Definition 3.11. A subset $A \subset X^{(\gamma, L)}$ is said to be weak " compact in the pair (X, $X^{(\gamma,}$ u) or, simply, weak "compact if $A$ is compact in $\left(\mathbb{K}^{X}\right.$, weak $\left.*\right)$, and $A$ is said to be relatively weak " compact in the pair $\left(X, X^{\gamma, U}\right)$ or, simply, relatively weak" compact if in $\left(\mathbb{K}^{X}\right.$, weak $\left.*\right)$ the closure $\bar{A}$ is compact and $\bar{A} \subset X^{(\gamma, U)}$.

For $A \subset X^{(\gamma, L)}, \bar{A}^{\text {weak* } *}$ stands for the closure of $A$ in $\left(\mathbb{K}^{X}\right.$, weak $\left.*\right)$. 
Theorem 3.12. For every $V \in \mathcal{N}(X), V^{*}=\left\{f \in X^{(\gamma, L)}:|f(x)| \leq 1, \forall x \in V\right\}$ is weak compact in the pair $\left(X, X^{(\gamma, U)}\right)$, and every equicontinuous $S \subset X^{(\gamma, L)}$ is relatively weak ${ }^{*}$ compact in the pair $\left(X, X^{(\gamma, L)}\right)$.

Proof. For each $x \in X$, let $x(f)=f(x)$ for $f \in \mathbb{K}^{X}$, then $x: \mathbb{K}^{X} \rightarrow \mathbb{K}$ is a linear functional. Let $V \in \mathcal{N}(X)$. By Corollary 3.6, $V$ is equicontinuous on $X$ and, by Lemma 3.2, $x\left(V^{*}\right)=\left\{f(x): f \in V^{*}\right\}$ is bounded in $\mathbb{K}$ for each $x \in X$, i.e., for each $x \in X, x\left(V^{*}\right)$ is totally bounded in $\mathbb{K}$ and so $V$ is totally bounded in $\left(\mathbb{K}^{X}, \omega x\right)$ for each $x \in X([[1], \mathrm{p}$. 84, Theorem 6]. But the weak" topology for $\mathbb{K}^{X}$ is just $\mathbf{V}\{\omega x: x \in X\}$ and so $V^{*}$ is totally bounded in $\left(\mathbb{K}^{X}\right.$, weak $\left.*\right)([[1]$, p. 85 , Theorem 7$]$.

Let $\left(f_{\alpha}\right)_{\alpha \in I} \subset V^{*}$ be a Cauchy net in $\left(\mathbb{K}^{X}\right.$, weak $\left.*\right)$. Then $\lim _{\alpha} f_{\alpha}(x)=f(x)$ exists at each $x \in X$ and so $f_{\alpha} \rightarrow f$ in $\left(\mathbb{K}^{X}\right.$, weak $\left.*\right)$. For $x \in X, u \in U$ and $t \in\{z \in \mathbb{K}:|z| \leq 1\}$,

$$
f(x+t u)=\lim _{\alpha} f_{\alpha}(x+t u)=\lim _{\alpha}\left[r_{\alpha} f_{\alpha}(x)+s_{\alpha} f_{\alpha}(u)\right], \text { where }\left|r_{\alpha}-1\right| \leq|\gamma(t)| \text { and }\left|s_{\alpha}\right| \leq|\gamma(t)|, \forall \alpha \in I .
$$

By passing to a subnet if necessary, we assume that $r_{\alpha} \rightarrow r$ and $s_{\alpha} \rightarrow s$ in $\mathbb{K}$. Then $\mid r$ $-1\left|=\lim _{\alpha}\right| r_{\alpha}-1|\leq| \gamma(t)|| s,\left|=\lim _{\alpha}\right| s_{\alpha}|\leq| \gamma(t)||$ and

$$
f(x+t u)=\lim _{\alpha}\left[r_{\alpha} f_{\alpha}(x)+s_{\alpha} f_{\alpha}(u)\right]=r f(x)+s f(u) .
$$

This shows that $f \in \mathcal{L}_{\gamma, U}(X, \mathbb{K})$.

Let $x_{\beta} \rightarrow x$ in $X$. Since $V$ is equicontinuous on $X$ and $f_{\alpha} \in V^{*}$ for all $\alpha \in I$, it follows from Lemma 3.9 that $\lim _{\beta} f_{\alpha}\left(x_{\beta}\right)=f_{\alpha}(x)$ uniformly for $\alpha \in I$. Then

$$
\lim _{\beta} f\left(x_{\beta}\right)=\lim _{\beta} \lim _{\alpha} f_{\alpha}\left(x_{\beta}\right)=\lim _{\alpha} \lim _{\beta} f_{\alpha}\left(x_{\beta}\right)=\lim _{\alpha} f_{\alpha}(x)=f(x)
$$

by Lemma 3.10, i.e., $f: X \rightarrow \mathbb{K}$ is continuous and hence $f \in X^{(\gamma, L)}$. Moreover, $|f(x)|=$ $\lim _{\alpha}\left|f_{\alpha}(x)\right| \leq 1$ for each $x \in V$, i.e., $f \in V^{*}$. Thus, $V^{*}$ is complete in $\left(\mathbb{K}^{X}\right.$, weak*). Since $\left(\mathbb{K}^{X}\right.$, weak $\left.*\right)$ is a topological vector space and $V^{*}$ is both totally bounded and complete in $\left(\mathbb{K}^{X}\right.$, weak $\left.*\right)$, we have $V^{*}$ is compact in $\left(\mathbb{K}^{X}\right.$, weak $\left.*\right)$, i.e., $V^{*}$ is weak* compact in the pair $\left(X, X^{(\gamma, L)}\right)$ ( [[1], p. 88, Theorem 7]).

Assume that $S \subset X^{(\gamma, L)}$ is equicontinuous on $X$. By Lemma 3.2,

$S^{\bullet}=\{x \in X:|f(x)| \leq 1, \forall f \in S\} \in \mathcal{N}(X)$, it follows from what is established above that $\left(S^{*}\right)^{\bullet}=\left\{f \in X^{(\gamma, U)}:|f(x)| \leq 1, \forall x \in S^{*}\right\}$ is compact in the Hausdorff space $\left(\mathbb{K}^{X}\right.$, weak $\left.*\right)$. Then $S \subset\left(S^{\bullet}\right)^{\bullet}$ shows that $\bar{S}^{\text {weak* }} \subset\left(S^{\bullet}\right)^{\bullet} \subset X^{(\gamma, U)}$ and $S$ is relatively weak* compact in $\left(X, X^{(\gamma, L)}\right)$.

Theorem 3.12 is a version of Alaoglu-Bourbaki theorem for the demi-linear dual pair $\left(X, X^{(\gamma, L)}\right)$, by which we can establish an improved Banach-Alaoglu theorem ( [[1], p. $130]$ as follows.

Corollary 3.13 (Banach-Alaoglu). Let $X$ be a seminormed space and $M>0$. Then

$$
S=\left\{f \in X^{(\gamma, U)}: \sup _{\|x\| \leq 1}|f(x)| \leq M\right\}
$$

is weak" compact in the pair $\left(X, X^{(\gamma, L)}\right)$.

Proof. Since $\sup _{f \in S} \sup _{|| x|| \leq 1}|f(x)| \leq M<+\infty$, Corollary 3.8 shows that $S$ is equicontinuous on $X$. By Theorem 3.12, $\bar{S}^{\text {weak } *} \subset X^{(\gamma, U)}$ and $\bar{S}^{\text {weak } *}$ is compact in $\left(\mathbb{K}^{X}\right.$, weak $\left.*\right)$. 
Let $\left(f_{\alpha}\right)_{\alpha \in I}$ be a net in $S$ such that $\lim _{\alpha} f_{\alpha}(x)=f(x)$ at each $x \in X$. Then $f \in X,(\gamma, L)$ and

$$
\sup _{\|x\| \leq 1}|f(x)|=\sup _{\|x\| \leq 1} \lim _{\alpha}\left|f_{\alpha}(x)\right| \leq M,
$$

i.e., $f \in S$. Thus, $\bar{S}^{\text {weak } *}=S$.

Theorem 3.14. Let $X$ be a separable space, $K$ a weak compact set in $X^{(\gamma, L)}, S$ an equicontinuous set in $X^{(\gamma, U)}$, and $V \in \mathcal{N}(X), V^{\bullet}=\left\{f \in X^{(\gamma, U)}:|f(x)| \leq 1, \forall x \in V\right\}$. Then $\left(S\right.$, weak*) is metrizable, and both $\left(K\right.$, weak* $\left.k^{*}\right)$ and $\left(V^{*}\right.$, weak $\left.k^{*}\right)$ are compact metric spaces.

Proof. Assume that $\left\{x_{n}\right\}_{n=1}^{\infty}$ is dense in $X$. Let

$$
d(f, g)=\sum_{n=1}^{\infty} \frac{1}{2^{n}} \frac{\left|f\left(x_{n}\right)-g\left(x_{n}\right)\right|}{1+\left|f\left(x_{n}\right)-g\left(x_{n}\right)\right|}, \forall f, g \in \mathbb{K}^{X} .
$$

Then, $d(\cdot, \cdot)$ is a pseudometric on $\mathbb{K}^{X}$. If $f, g \in X^{(\gamma, L)}$ and $d(f, g)=0$, then $f\left(x_{n}\right)=g\left(x_{n}\right)$ for all $n$. Since both $f$ and $g$ are continuous on $X$ and $\left\{x_{n}\right\}_{n=1}^{\infty}$ is dense in $X, f(x)=g(x)$ for all $x \in X$, i.e., $f=g$. This shows that $\left(X^{(\gamma, L)}, d\right)$ is a metric space, and $f_{k} \rightarrow f$ in $\left(X^{(\gamma,}\right.$ $\left.{ }^{u)}, d\right)$ if and only if $\lim _{k} f_{k}\left(x_{n}\right)=f\left(x_{n}\right)$ for each $n \in \mathbb{N}$. Hence, weak* is stronger than $d$ $(\cdot, \cdot)$ and so the compact space (K, weak* $)$ is homeomorphic to the (Hausdorff) metric space $(K, d)$. Thus, $\left(K\right.$, weak $\left.k^{*}\right)$ is a compact metric space.

By Theorem 3.12, in $\left(\mathbb{K}^{X}\right.$, weak $\left.*\right)$ the closure $\bar{S}^{\text {weak } *} \subset X^{(\gamma, U)}$, and both $\left(\bar{S}^{\text {weak* } *}\right.$, weak $\left.*\right)$ and $\left(V *\right.$ weak $\left.k^{*}\right)$ are compact and so they are compact metric spaces.

The following special case of Theorem 3.14 is a well-known fact ([[1], p. 143]).

Corollary 3.15. Let $X$ be a separable locally convex space with the dual $X, K$ a weak" compact set in $X^{\prime}, S$ an equicontinuous set in $X^{\prime}$, and $V \in \mathcal{N}(X), V^{\circ}=\left\{f \in X^{0}: \mid\right.$ $f(x) \mid \leq 1, \forall x \in V\}$. Then $\left(S\right.$, weak $\left.k^{*}\right)$ is metrizable, and both $\left(K\right.$, weak* $\left.k^{*}\right)$ and $\left(V^{\circ}\right.$, weak $\left.k^{*}\right)$ are compact metric spaces.

Corollary 3.16. Let $X$ be a separable space and $S$ an equicontinuous set in $X^{(\gamma, U)}$. Every sequence $\left\{f_{n}\right\}$ in $S$ has a subsequence $\left\{f_{n_{k}}\right\}$ such that $\lim _{k} f_{n_{k}}(x)=f(x)$ exists at each $X \in X$ and the limit function $f \in X^{(\gamma, L)}$, i.e., $f$ is both continuous and demi-linear.

Proof. By Theorems 3.12 and 3.14, $\bar{S}^{\text {weak } *} \subset X^{(\gamma, U)}$ and $\left(\bar{S}^{\text {weak* } *}\right.$, weak*) is a compact metric space. Then $\left(\bar{S}^{\text {weak } *}\right.$, weak $\left.*\right)$ is sequentially compact.

Combining Theorem 1.4 and Corollary 3.16, we have the following

Corollary 3.17. Assume that $X$ is of second category and separable, e.g., separable Fréchet spaces such as $L^{p}(0,1)(p>0), C[0,1], c_{0}, c, l^{p}(p>0)$, etc. If $S \subset X^{(\gamma, L)}$ is pointwise bounded on $X$, then every sequence $\left\{f_{n}\right\}$ in $S$ has a subsequence $\left\{f_{n_{k}}\right\}$ such that $\lim _{k} f_{n_{k}}(x)=f(x)$ exists at each $x \in X$, and $f \in X^{(\gamma, L)}$.

For $C \geq 1$ and $\delta>0$, letting $\gamma(t)=C t$ for $t \in \mathbb{R}$ and $U=(-\delta, \delta)$, we have $\gamma \in C(0)$ and $U \in \mathcal{N}(\mathbb{R})$. Then let $\mathbb{R}^{(C, \delta)}=\mathbb{R}^{(\gamma, U)}$. It is easy to see that every $f \in \mathcal{L}_{\gamma, U}(\mathbb{R}, \mathbb{R})$ is continuous and so $\mathbb{R}^{(C, \delta)}=\mathcal{L}_{\gamma, U}(\mathbb{R}, \mathbb{R})$. Thus, $\mathbb{R}^{(C, \delta)}$ contains all linear functions and various nonlinear functions. It is noted that many functions in $\mathbb{R}^{(C, \delta)}$ have very complicated graphs.

For $S \subset \mathbb{R}^{(C, \delta)}$, there is an interesting fact: a local behavior in a small interval $(-\varepsilon, \varepsilon)$ implies a nice behavior on $(-\infty,+\infty)$. 
Example 3.18. Let $S \subset \mathbb{R}^{(C, \delta)}$. If there exists $M, \varepsilon>0$ such that $|f(x)| \leq M$ for every $f$ $\in S$ and $x \in(-\varepsilon, \varepsilon)$, then every $\left\{f_{n}\right\} \subset S$ has a subsequence $\left\{f_{n_{k}}\right\}$ such that $\lim _{k} f_{n_{k}}(x)=f(x)$ exists at each $x \in \mathbb{R}$, and $f \in \mathbb{R}^{(C, \delta)}$.

In fact, $\mathbb{R}$ is separable and $(-\varepsilon, \varepsilon) \in \mathcal{N}(\mathbb{R})$. The assumption shows that

$$
M^{-1} S \subset\left\{f \in \mathbb{R}^{(C, \delta)}:|f(x)| \leq 1, \forall x \in(-\varepsilon, \varepsilon)\right\}=(-\varepsilon, \varepsilon)^{\bullet}
$$

By Theorem 3.14, $\left((-\varepsilon, \varepsilon)^{\bullet}\right.$, weak* $k^{*}$ is a compact metric space and so it is sequentially compact. Similarly, we have

Example 3.19. Let $p>0$ and $S \subset\left(L^{p}(0,1)\right)^{(\gamma, U)}$. If there exists $\varepsilon>0$ such that $|f(x)| \leq$ 1 whenever $f \in S$ and $X \in L^{p}(0,1)$ with $\|x\|<\varepsilon$, then every $\left\{f_{n}\right\} \subset S$ has a subsequence $\left\{f_{n_{k}}\right\}$ such that $\lim _{k} f_{n_{k}}(x)=f(x)$ exists for all $x \in L^{p}(0,1)$, and $f \in\left(L^{p}(0,1)\right)^{(\gamma, U)}$.

We shall show that the condition "sup $f \in S,|| x||<\varepsilon|f(x)| \leq 1$ " in Example 3.19 can be weakened as " $\sup _{f \in S}|f(x)|<+\infty, \forall\|x\|<\varepsilon$ " (see Corollary 3.20).

In general, combining Theorems 3.12 and 3.14, we have

Corollary 3.20. Let $S \subset X^{(\gamma, U)}$. If there exists $V \in \mathcal{N}(X)$ such that $\sup _{f \in S, x \in V}|f(x)|<$ $+\infty$, then

(a) $S$ is equicontinuous on $X$,

(b) $S$ is relatively weak * compact,

(c) every net $\left(f_{\alpha}\right)$ in $S$ has a subnet $\left(f_{\xi}(\alpha)\right)$ such that $\lim _{\xi(\alpha)} f_{\xi}(\alpha)(x)=f(x)$ exists for all $x \in X$, and $f \in X^{(\gamma, U)}$.

If, in addition, $x$ is separable, then

(d) every $\left\{f_{n}\right\} \subset S$ has a subsequence $\left\{f_{n_{k}}\right\}$ such that $\lim _{k} f_{n_{k}}(x)=f(x)$ exists for all $x$ $\in X$, and $f \in X^{(\gamma, U)}$.

In fact, for $M=\sup _{f \in S, x \in V}|f(x)|$, we have $A=\left\{\frac{1}{M+1} f: f \in S\right\} \subset V^{\bullet}$ and (a)-(d) hold for $A$, i.e., $S$ satisfies (a)-(d).

If $X$ is of second category, then the condition "there exists $V \in \mathcal{N}(X)$ such that sup$f \in S, x \in V|f(x)|<+\infty$ " in Corollary 3.20 can be weakened as "there exists $V \in \mathcal{N}(X)$ such that $\sup f \in S|f(x)|<+\infty, \forall x \in V^{\prime \prime}$.

To see this, we first establish a simple fact.

Lemma 3.21. Let $\Gamma \subset \mathcal{L}_{\gamma, U}(X, Y)$. If there exists $V \in \mathcal{N}(X)$ such that $\{f(x): f \in \Gamma\}$ is bounded at each $x \in V$, then $\{f(x): f \in \Gamma\}$ is bounded at each $x \in X$.

Proof. Let $x \in X$. There exists $n_{0} \in \mathbb{N}$ such that $\frac{1}{n_{0}} x \in V \cap U$. By Lemma 3.1, for each $f \in \Gamma$, we have

$$
f(x)=f\left(n_{0} \frac{x}{n_{0}}\right)=\alpha_{f} f\left(\frac{x}{n_{0}}\right), \quad \text { where }\left|\alpha_{f}\right| \leq 2(1+|\gamma(1)|)^{n_{0}-1}-1 .
$$

Then

$$
\{f(x): f \in \Gamma\} \subset\left\{t f\left(\frac{x}{n_{0}}\right): f \in \Gamma,|t| \leq 2(1+|\gamma(1)|)^{n_{0}-1}-1\right\} .
$$


Since $\quad \frac{x}{n_{0}} \in V, \quad\left\{f\left(\frac{x}{n_{0}}\right): f \in \Gamma\right\} \quad$ is $\quad$ bounded $\quad$ and so $\left\{t f\left(\frac{x}{n_{0}}\right): f \in \Gamma,|t| \leq 2(1+|\gamma(1)|)^{n_{0}-1}-1\right\}$ is bounded.

Now we can improve Theorems 1.4 and 1.5 as follows.

Theorem 3.22. Assume that $x$ is of second category and $\Gamma \subset\left\{f \in \mathcal{L}_{\gamma, U}(X, Y): f\right.$ is continuous $\}$. If there exists $V \in \mathcal{N}(X)$ such that $\Gamma$ is pointwise bounded on $V$, then $\Gamma$ is equicontinuous on $X$, and $\Gamma$ is uniformly bounded on each bounded subset of $X$.

Corollary 3.23. Assume that $x$ is of second category and $S \subset X^{(\gamma, U)}$. If there exists $V \in \mathcal{N}(X)$ such that $\sup _{f \in S}|f(x)|<+\infty$ at each $x \in V$, then (a)-(c) hold for $S$. If, in addition, $X$ is separable, then (d) holds for $S$.

We now show that every equicontinuous $S \subset X^{(\gamma, U)}$ has a nice behavior on any compact subset of $X$.

Theorem 3.24. Let $X$ be a Hausdorff topological vector space. If $S$ is an equicontinuous subset of $X^{(\gamma, U)}$ and $\Omega$ is a compact subset of $X$, then every $\left\{f_{n}\right\} \subset S$ has a subsequence $\left\{f_{n_{k}}\right\}$ such that $\lim _{k} f_{n_{k}}(x)=f(x)$ uniformly for $x \in \Omega$ and $f: f: \Omega \rightarrow \mathbb{K}$ is continuous.

Proof. Let $K=\left\{\left.f\right|_{\Omega}: f \in S\right\}$. Then $K \subset C(\Omega)$ and $K$ is equicontinuous at each $x \in \Omega$. Suppose that $\sup _{f \in K}|| f \|_{\infty}=\sup _{f \in K, x \in \Omega}|f(x)|=+\infty$. Then there exist sequences $\left\{f_{n}\right\} \subset$ $S$ and $\left\{x_{n}\right\} \subset \Omega$ such that $\left|f_{n}\left(x_{n}\right)\right|>n, \forall n \in \mathbb{N}$. By Lemma 3.2, we may assume that $x_{n}$ $\neq x_{m}$ for $n \neq m$.

Since $\Omega$ is compact, $\left\{x_{n}\right\}_{n=1}^{\infty}$ has a cluster point $x \in \Omega$.

Since $S$ is equicontinuous at $x$, there exists $V \in \mathcal{N}(X)$ such that $|f(y)-f(x)|<1$ for all $f \in S$ and $y \in x+V$, i.e., $|f(y)|<|f(x)|+1$ for all $f \in S$ and $y \in x+V$. Observing that $\left|f_{n}\left(x_{n}\right)\right|>n$ for all $n \in \mathbb{N}$ and $\left\{f_{n}\right\} \subset S$, there exists $n_{0} \in \mathbb{N}$ such that $x_{n} \notin x+V$ for all $n>n_{0}$. Since $(x+V) \cap \Omega$ contains some $x_{n}$ with $x_{n} \neq x$, it follows that

$$
\emptyset \neq\left(\left\{x_{n}: x_{n} \neq x, x_{n} \in(x+V)\right\} \cap \Omega\right) \subseteq\left\{x_{n} \neq x: n \leq n_{0}\right\}=\left\{y_{1}, y_{2}, \cdots, y_{m}\right\},
$$

where $m \leq n_{0}$. But $X$ is Hausdorff, so $\Omega$ is also Hausdorff. Then there exists $V_{0} \in \mathcal{N}(X)$ such that $V_{0} \otimes V$ and $\left(x+V_{0}\right) \cap\left(\Omega \cap\left\{y_{1}, y_{2}, \cdots, y_{m}\right\}\right)=\varnothing$. Hence $x_{n} \in(x$ $\left.+V_{0}\right) \cap \Omega$ implies that $x_{n}=x$.

This contradicts the fact that $x$ is a cluster point of $\left\{x_{n}\right\}_{n=1}^{\infty}$. Hence, $\sup _{f \in \mathbb{K}} \| f||_{\infty}<+\infty$

By the Arzela-Ascoli theorem, $K$ is relatively compact in the metric space $(C(\Omega)$, $\left.\|\cdot\|_{\infty}\right)$. Hence, every $\left\{f_{n}\right\} \subset S$ has a subsequence $\left\{f_{n_{k}}\right\}$ such that $\left\|\left.f_{n_{k}}\right|_{\Omega}-f\right\|_{\infty} \rightarrow 0$, where $f \in C(\Omega)$, i.e., $\lim _{k} f_{n_{k}}(x)=f(x)$ uniformly for $x \in \Omega$.

Corollary 3.25. Let $X=\mathbb{R}^{n}$ or $\mathbb{C}^{n}, \varepsilon>0$ and $D_{m}=\{x \in X:\|x\| \leq m \varepsilon\}, \forall m \in \mathbb{N}$. If $S$ $\subset X^{(\gamma, U)}$ is pointwise bounded on $D_{1}$, then every sequence $\left\{f_{k}\right\} \subset S$ has a subsequence $\left\{f_{k_{i}}\right\}$ such that $\lim _{i} f_{k_{i}}(x)=f(x)$ uniformly on each $D_{m}$, where $f \in X^{(\gamma, U)}$.

Proof. Theorem 3.22 shows that $S$ is equicontinuous on $X$ and, by Theorem 3.24, $\left\{f_{k}\right\}$ has a subsequence $\left\{f_{k_{i}}\right\}$ such that $\lim _{i} f_{k_{i}}(x)$ exists uniformly on $D_{1}$. Then $\left\{f_{k_{i}}\right\}_{i=2}^{\infty}$ has a subsequence $\left\{f_{k_{i v}}\right\}$ such $\lim _{v} f_{k_{i v}}(x)$ exists uniformly on $D_{2}$. Proceeding inductively, the diagonal procedure yields a subsequence $\left\{g_{i}\right\}$ of $\left\{f_{k}\right\}$ such that $\lim _{i} g_{i}(x)$ exists uniformly 
on each $D_{m}$. Then $\lim _{i} g_{i}(x)=f(x)$ exists at each $x \in X$ and $f \in \bar{S}^{\text {weak* } *}$ in $\left(\mathbb{K}^{X}\right.$, weak* $)$. By Theorem 3.12, $f \in X^{(\gamma, U)}$.

\section{Author details}

${ }^{1}$ Department of Mathematics, Harbin Institute of Technology, Harbin 150001, P.R. China ${ }^{2}$ Department of Mathematics, Tianjin University, Tianjin 300072, P.R. China

\section{Authors' contributions}

RL gave the basic ideas and composed the main skeleton of this paper. His work includes the main theorems in section 2 and 3, and some concrete examples. AC provided more examples in section 2, proved some corollaries in section 2, 3, and drafted the manuscript. SZ participated in the discussion of the ideas and provided some insightful suggestion. All authors read and approved the final manuscript.

\section{Competing interests}

The authors declare that they have no competing interests.

Received: 9 June 2011 Accepted: 2 December 2011 Published: 2 December 2011

\section{References}

1. Wilansky, A: Modern Methods in Topological Vector Spaces. McGraw-Hill, New York (1978)

2. Li, R, Zhong, S, Li, L: Demi-linear analysis I-basic principles. J Korean Math Soc. 46(3), 643-656 (2009). doi:10.4134/ JKMS.2009.46.3.643

3. Khaleelulla, SM: Counterexamples in Topological Vector Spaces. Springer, New York (1982)

4. Iyahen, SO: Semiconvex spaces. Glasg Math J. 9, 111-118 (1968). doi:10.1017/50017089500000380

5. Köthe, G: Topological Vector Spaces I. Springer, New York (1969)

6. Dunford, N, Schwartz, J: Interscience, New York (1958)

doi:10.1186/1029-242X-2011-128

Cite this article as: Li et al.: Demi-linear duality. Journal of Inequalities and Applications 2011 2011:128.

\section{Submit your manuscript to a SpringerOpen ${ }^{\odot}$} journal and benefit from:

- Convenient online submission

- Rigorous peer review

- Immediate publication on acceptance

- Open access: articles freely available online

- High visibility within the field

- Retaining the copyright to your article

Submit your next manuscript at $\gg$ springeropen.com 